\title{
Fernand Hörner (Hg.): Kulturkritik und das Populäre in der Musik. Münster/New York, Waxmann, 2016. ISBN 978-3-8309-3361-8.
}

\section{Seiten.}

„Populäre Musik ist ein allgegenwärtiges Phänomen“ (7), so der erste Satz des vorliegenden Sammelbandes, und doch geht es in ihm nicht nur um , populäre' Musik, sondern mindestens so sehr um ,das Populäre' in der Musik, das auch in der sogenannten E-Musik seinen Platz finden kann. Dieses ,Populäre', in der Folge auf ,populäre` Musik beschränkt, soll laut Herausgeber nicht „essentialistisch“ (7) definiert werden, sondern Gegenstand einer problematisierenden Erörterung sein, die einen breiten Fächer von sich zum Teil überschneidenden Fragestellungen aufspannt und „Konstanten, unter wechselnden Vorzeichen“ (7) aufspürt. Reprisen sind dabei unvermeidlich, wie F. Hörner selbst bemerkt, so wenn beispielsweise in Teil 3 die Frage, „wie und wo das ästhetische Urteil über populäre Musik in Vermengung mit ethischen Überlegungen in die Kulturkritik gelangt" (11), erneut aufgegriffen wird.

Gewiss, statt dieser definitorischen Verweigerung hätte man mit Ch. Jost (2012) die populäre Musik als „Gesamtheit jener musikkulturellen Praktiken, deren Entstehung bis in die bürgerlichen Gesellschaften (und urbanen Zentren) des 19. Jahrhunderts zurückverfolgt werden kann und deren Entwicklung wesentlich durch massenkommunikative Medienpraktiken beeinflusst wurde," definieren können oder mit der IASPM nicht etwa als einen musikalischen Stil, sondern als „una galassia di musiche comprendente un vasto insieme di stili e generi circolanti attraverso i media e fruiti da un pubblico di massa. Ciò vuol dire ad esempio rock, pop, punk, rap e canzone d'autore, ma anche world music, musica per cinema e televisione, e persino musiche ,classiche ${ }^{\text {ed }}$,etniche riciclate dal sistema dei media“ (http://www.iaspmitalia.net/cose-la-iaspm/). Dennoch: Es ist richtig, dass eine „normative Definition des Populären und insbesondere der populären Musik“ (8) an die Quadratur des Kreises erinnert, und es ist auch richtig, dass der Band als Ganzes gelesen - mit seinen unzähligen netzartigen Verweisen und den Versuchen zumindest einzelner Verfasser, ihr Objekt nach Funktion, Aufführungsort, Geschmack etc. (cf. K. Maase) zu präzisieren - gerade dadurch beeindruckt, dass er dem Leser die Komplexität und inhaltliche Diversifizierung dessen, was man gemeinhin ,populäre Musik‘ oder auch „das ,Populäre` an der populären Musik“ (7) nennt, vor Augen führt.

Um dieses enorme Spektrum zu gliedern, greift F. Hörner auf M. Foucaults Überlegungen zur „Formation der Objekte“ aus der Archäologie des Wissens (1969) zurück und geht in vier Abschnitten mit den Untertiteln „1. Das Objekt: Populäre und andere Musik“, „2. Die Akteure: Produzenten und Rezipienten“, „3. Die Begriffe: Kulturkritische Theorie“ und „4. Die Strategien: Ästhetik und Ethik“ den Leitfragen nach, „(1) wie kulturkritische 
Diskurse, durch Abgrenzung und Eingrenzung, ,populäre Musik' als Objekt der Kritik festlegen, (2) wie sich die Diskursträger zum Objekt populäre Musik positionieren, (3) auf welche Begriffe und Konzepte sie zurückgreifen und (4) wie sie diese strategisch verwenden, kombinieren und gegeneinander ausspielen" (8). Die 16 Beiträge basieren auf einer diskurskritischen Position, d.h. dass die diversen Diskursträger den Gegenstand, von dem sie in der Folge sprechen, selbst produzieren. Interessant sind auch die zahlreichen Diskursüberlagerungen, die insbesondere in Abschnitt 4 zur Sprache kommen (ästhetischer, ethischer, theologischer, ethnologischer Diskurs etc.), wobei sich das Diskurspaar ästhetisch/ ethisch generell als eine jener Konstanten entpuppt, von denen der Verfasser im Vorwort spricht.

Der zweite Schlüsselbegriff des Buches, ,Kulturkritik', wird gleich zu Beginn des ersten Abschnitts erörtert, und es ist das Verdienst von Th. Hecken, dass er den Leser geschickt in die historischen Konnotationen und ideologischen Aufladungen eines Terminus einführt, den G. Bollenbeck zunächst neutral als „Klagen über den allgemeinen Verfall der Sitten und der Gesellschaft, über Entfremdung und Rationalisierung, über die unheilvolle Herrschaft des Geldes, der Technik oder der Medien“ (17) umschrieben hatte. Hecken zeigt Berührungspunkte und Differenzen zwischen dem Kulturpessimismus der (vorletzten) Jahrhundertwende, dem Nationalsozialismus affinen Standpunkten und der Kritik an der Massengesellschaft der Nachkriegszeit; zugleich lassen seine Ausführungen zur Bewertung moderner populärer Kultur aber auch eine gewisse Nähe zwischen „neulinke[n] Gesellschafts- und rechte[n] Kulturkritikern" erkennen und die Haltung von Th. W. Adorno und M. Horkheimer in einem neuen Licht erscheinen. Mit der neuen „Popaffirmation“ (28) der 1990er Jahre, so Hecken, verschwinden kultur- und/oder gesellschaftskritische Positionen zwar vorübergehend, doch bald schon macht sich eine neue Dichotomie bemerkbar, die zwischen „[g]latte[m]“, „[n]arzisstische[m]“ Pop (29) und dem „große[...] Gemeinschaftserlebnisse“ (29) gewährenden kreativen Rock - später Underground, Alternative, Independent und wie immer - unterscheidet.

Vor dem derart abgesteckten Rahmen widmen sich die folgenden drei Beiträge dem Objekt ,Populäre Musik' unter jeweils unterschiedlichen Vorzeichen, doch stets unter Berücksichtigung einer historischen Perspektive: Populäre Musik stoße bei ihren Kritikern auf weniger soziale Ablehnung als etwa , triviale 'Literatur, schließt K. Maase aus seiner rezeptionsästhetischen Studie zum Kampf gegen „Schmutz und Schund“ (35) in den vergangenen 120 Jahren und führt dies u.a. auf das geringere „narrative[...] Potenzial" (44) der populären Musik und ihre ,ungefährliche' Funktion als „alltäglicher Stimmungsgenerator" (47) zurück. D. B. Scott spürt den Grenzen zwischen Kunst und Unterhaltung nach und gibt unter Bezugnahme auf Hanslick, dann auf C. Dahlhaus, Th. W. Adorno und P. Bourdieu einen erhellenden Einblick in die diversen Versuche der Abgrenzung von populärer Musik als ,Trivialmusik', ,Kitsch', ,leichte Musik', ,affirmative Musik' bis hin zur ,U-Musik'. W. Jansen nähert sich dem Objekt nicht von der Musik, sondern vom Theater her und stellt der genannten Begriffsvielfalt das, Tingeltangel' an die Seite, das - bald schon mit einem verderblich offenen Umgang mit Sexualität assoziiert - zwar 1869 von der gesetzlichen The- 
aterreform noch profitiert, jedoch ab 1880 und definitiv ab 1883 der neuen „Zwei-KlassenGesetzgebung" (79) im Bereich des Theaters zum Opfer fällt.

Dem Thema des Akteurs - oder ,wie sich die Diskursträger zum Objekt populäre Musik positionieren" (8) - sind die vier Beiträge von Abschnitt 2 gewidmet. Sie setzen sich auf intensive und überaus produktive Weise mit Bourdieus Distinktionstheorie und deren kulturkritischem Potential (9) auseinander, so wie Abschnitt 3 - „Die Begriffe“ - die Zusammenführung von Adornos „theoretischen Überlegungen zum dialektischen Verhältnis von Kultur und Kritik mit seiner eigenen ,praktischen' Kulturkritik" (10-11) beinhaltet. Die Zuordnung der acht Beiträge zu „Akteuren“ und „Begriffen“ mag daher etwas gewollt erscheinen, denn im Grunde geht es einmal im Wesentlichen um Bourdieu und einmal um Adorno, die F. Hörner zu Beginn seiner Studie „Seine Unterschiede. Kulturkritische Ansätze bei Bourdieu" miteinander vergleicht. Ungeachtet Bourdieus kritischer Haltung gegenüber der Frankfurter Schule sieht Hörner das Gemeinsame im Interesse beider für das Objekt ,populäre Musik', für die Rolle der Kultur in der Gesellschaft, die Ökonomie der Kultur und die verborgenen Machtverhältnisse, wobei bei Adorno die Uniformierung durch die Kulturindustrie, bei Bourdieu das Distinktionspotential von Kultur auf der Ebene von Produzenten und Rezipienten im Mittelpunkt steht. Adorno wie Bourdieu unterscheiden zwischen zwei Ebenen von Kultur, Bourdieus Standpunkt könne jedoch als ein eher relationaler beschrieben werden. Umgekehrt weist Hörner kritisch darauf hin, dass in Bourdieus Die feinen Unterschiede (2011) populäre Kultur zu wenig ausdifferenziert gesehen wird. In seinem grundlegenden Beitrag geht es ihm daher darum, Bourdieus „Konzepte des Populären" (86) freizulegen (Kultur, Klasse, Künstler, Geschmack) und ihr Zusammenspiel auf ihre Anwendbarkeit zu überprüfen. Dabei erläutert er den Bourdieu'schen Kapitalbegriff, die Homologien „von Sozialraum und ästhetischen Vorlieben“, von „Rezipienten und Konsumenten“" und „von Kulturgütern bzw. Klassenlage und dem dazu ,passendem“ [sic] Geschmack“ (90) - letzteres eine Homologie, die eine Differenzierung „zwischen legitimer, mittlerer und populärer Kultur" voraussetzt. Die Differenzierung von legitimem (also hegemonialem) und populärem Geschmack hingegen - und hier kommt das System in Bewegung - erweist sich als ,nicht homolog übertragbar' auf die Unterscheidung von legitimer und populärer Kultur, sondern kann ,jeweils sowohl auf legitime als auch auf populäre Musik angewendet werden" (109).

Wie die Arbeit von Hörner, so würden auch die drei weiteren Beiträge des Abschnitts 2 eine weitaus detailliertere Besprechung verdienen, als hier möglich ist. Notieren wir kursorisch zumindest die wichtigsten Thesen: N. Geer geht in die postmodernen 1980er Jahre, wenn sie sich mit der bundesdeutschen Popkritik, dem Popkritiker unter dem Gesichtspunkt der „Sophistication“ (117) beschäftigt, einem „Denkstil [...], der eng an den deutschen Popdiskurs gekoppelt war“, ja zwischen Mainstream und Linksalternative als „Habitus“ der deutschen „Popintelligenz“ (117) gelten konnte. Als eng verbunden damit sieht Geer die Pose, „die selbstreflexive ,Als-Ob-Authentizität‘ des Pop als ,Authentizität zweiter Ordnung “" (124), an der sich jede Popkritik abarbeiten muss. Ist die bundesdeutsche Popkritik somit hierarchisch oder antihierarchisch, ist sie revolutionär oder elitär, ist sie kontemporär 
oder aber ist sie einfach ein Fake? Alles Fragen, die bewusst auf die Ambiguität des Feldes und insbesondere auf die des Kritikers aufmerksam machen. M. Parzer beleuchtet in seiner Untersuchung das „kulturkritische Potential der Allesfresser-These“ (135) nach Bourdieu. Erneut ist der Fokus auf der Zeit ab den 1980er Jahren, der Parzer „zunehmend eine [...] positive [...] Bewertung populärkultureller Formen" (135) attestiert, welche sich u.a. im Phänomen des ,Allesfressers" und seinem „breit gefächerten“ und „grenzüberschreitenden Geschmack" (136) niederschlägt. In einer glasklaren und stringenten Replik auf Die feinen Unterschiede thematisiert er die die Geschmacksbildung beeinflussenden und sich verändernden gesellschaftlichen Faktoren, die dazu führen, dass eine „Vorliebe für ,klassische Musik“ nicht mehr mit sozialem „Distinktionsgewinn“ (139) korrelieren muss. Heute gehe es nicht darum, „an welchen, sondern an wie vielen unterschiedlichen Genres“ (140) ein Rezipient Gefallen findet. Ist dies ein Zeichen von Offenheit und Toleranz? Der Verfasser tendiert zu einem Nein, denn selbst wenn ,kulturelles Kapital' heute nicht mehr die Zwangsehe mit der Hochkultur, sondern vielmehr den „flexible[n] und kompetente[n] Umgang mit unterschiedlichen Ästhetiken" (146-147) bedeutet, haben nicht alle Rezipienten dieselbe Chance, einen „grenzüberschreitende[n] Musikgeschmack“ (148) zu entwickeln. Um eine andere Art der Konsekration populärer Musik geht es schließlich in J. Kropfs Studie des musikalischen Felds. In seinen bestens dokumentierten Ausführungen zu Popmusikjournalismus, Feuilleton und Bildungswesen konstatiert er neben einer generellen, in den USA stärker als in Deutschland spürbaren Aufwertung der populären Musik zugleich und gegenläufig den Rekurs auf traditionelle, ja , hochkulturelle‘ Beurteilungskriterien, die zeigen, dass Bourdieus Thesen noch keineswegs gänzlich überholt sind.

Was Adorno betrifft, so beginnt Abschnitt 3 mit einem referenzreichen Beitrag von H.-E. Schiller zu Adornos Überlegungen zur Kulturkritik, im Zuge dessen zunächst ,Kultur' ins Visier genommen wird: Kultur im Sinn von „bürgerlicher Geisteskultur“ des 18. Jahrhundert mit Wissenschaft, Kunst und Philosophie als integrativen Komponenten, Kultur dann später im Sinne von Horkheimer, der „neben Kunst, Religion und Philosophie (die Stufen des absoluten Geistes nach Hegel) auch die Lebensweise, Gewohnheiten, Sitten“ (187) mit einbezieht. Schiller diskutiert des Weiteren die für Adorno problematischen Implikationen der ,Kulturkritik', um letztendlich ausgehend vom Begriff des ,Kulturverfalls' einen Blick auf Adornos Verständnis von ,Kulturindustrie' (Warencharakter, Fetisch) und die bekannten Zuschreibungen der Popmusik (Unmündigkeit, Zerstreuung, Unaufmerksamkeit, Abfolge äußerer Reize etc.) zu werfen. Im Zentrum des Beitrags von U. Plass stehen Adornos überaus polemische Jazz-Kritiken aus den Jahren 1932 bis 1953, in denen temporale Aspekte wie Zeitlichkeit, Geschichtlichkeit oder das ,Zufrühkommen' als Leitfaden dienen. In einem trotz der Komplexität der Materie stets lesbaren Artikel entwickelt Plass ein Dreistufenmodell nach Adorno, das den Jazz zunächst (1933) ,als historisch obsolet, weil ästhetisch verbraucht“ (220) deklariert, ihn sodann (1936) „mit Hilfe der Theorie des Zufrühkommens als sadomasochistisches Phänomen“ unter „Faschismus-Verdacht" einstuft und schließlich 1953 „am Anfang einer der ästhetisch wichtigsten Dekaden avantgardistischer Jazz-Musik“ zu einer „zeitlosen“ Mode „entzeitlicht und enthistorisiert“ (220). Konstanten in diesem 
Prozess sind die Betonung des Warencharakters und die Gleichsetzung des Jazz mit der geschichtslosen und genormten populären Musik der Kulturindustrie sowie die Vernachlässigung sowohl einer differenzierten ,immanenten' musikalischen Analyse als auch der dem Jazz zugrundeliegenden „spezifisch [afro]amerikanische[n] Musik“ (196-197). Adorno, so Plass und andere, verkenne die dem Jazz ursprünglich inhärente Spannung zwischen Normierung und Subjektivität und die Tatsache, dass die immer gleichen strukturellen musikalischen Elemente nicht statischer „Schein rhythmischer Freiheit“ (223) sein müssen, sondern in der „intensive[n] Konzentration auf den Moment“ (223) zu einer neuen Zeitlichkeit führen. Eine ähnlich kritische Haltung nimmt A. Gayraud ein, die dem Adorno’schen (Vor)Urteil nachgeht, populäre Musik eigne sich nicht für ein ,aktives' Hören. Nach einem Blick auf Adornos Typologie des Hörers, die sich zwischen dem „Experten-Hörer“ und dem mit den Stigmata der Unterhaltung und Bequemlichkeit behafteten „musikalisch Gleichgültige[n]“, „Unmusikalische[n]“, ja „Antimusikalischen“ (228) bewegt, postuliert sie, dass auch populäre Musik die nötigen Voraussetzungen für ein strukturelles Hören - nämlich „both extremes of the dialectic: the singular detail and the whole" (232) zu beinhalten - bieten kann. Als Weg dahin benennt sie fünf Schritte (233-236), die der Hörer/Kritiker von populärer Musik zu berücksichtigen habe: eine revidierte Auffassung des „aesthetic material“ der Popmusik und ihres „sound treatment“, die Anerkennung des für die Popmusikanalyse nötigen Wissens sowie die ihrer Metaebene, i.e. der „pop music's self-consciousness of its own authenticity“, und schließlich die Anerkennung ihres „stunning effect“, der keinesfalls mit dem passiven Hören zusammenfallen muss. Ein aktives, selektives und langsames Hören von Popmusik werde nicht zuletzt im Zeitalter des Internet, des Streaming etc. zu einer Frage der Verantwortung, ja einer Art ethischer Verpflichtung. Auch der kulturhistorisch besonders spannende Beitrag von Ch. Jost, der den Schwerpunkt auf die „Anfänge [...] der didaktischen Konzeptualisierung populärer Musik in Deutschland“ (241) legt, geht in eine ähnliche Richtung. Wenn in der Nachkriegszeit die konservative (auch restaurative) Kulturkritik zu einem Bildungsprogramm werden konnte, das die „technisch-medial[e] Durchdringung des Alltags" (245) ablehnt und sich in diesem Punkt paradoxerweise mit Adornos ,linker' Kritik der kapitalistischen Ideologie berührt, so verdankt die Musikdidaktik gerade Adornos systematischer Analyse und seinem Insistieren auf den „Funktionen, Strukturen und Wirkungen populärer Musik“ (257) einen „didaktische[n] Quantensprung“ (252). Die Behandlung der Massenkultur im schulischen Raum wird somit legitimiert, ja zu einem bildungspolitischen Auftrag.

Nach diesem sehr runden 3. Abschnitt zu Adorno verlagert sich der Blick im letzten Teil, unter erneuter Fokussierung der Konstanten „Ästhetik und Ethik“, in Richtung „Strategien“. Jeder der vier abschließenden Beiträge verbreitert das thematische Spektrum des Buches ganz entscheidend und gibt - vom Leser mit Spannung erwartet - Hinweise auf die praktischen Auswirkungen der so breit diskutierten ästhetisch-ethischen Positionen und Begrifflichkeiten. Auf die Interdiskursivität dieses Abschnitts wurde bereits eingangs verwiesen und so überrascht es auch nicht, wenn gleich die erste Studie von M. Fischer der Frage „Pop and Pope. Affirmation und Negation des Populären im Katholizismus“ gewidmet ist. 
Der Titel ist dabei zugleich Resümee: Die generelle Ablehnung der Moderne wie auch der Postmoderne, die „Grenzziehungen zwischen richtig und falsch“ (279) negiert, äußert sich in den mehr als heftigen verbalen Attacken von Kardinal Joseph Ratzinger, dem späteren Papst Benedikt XVI., während umgekehrt andere hohe Würdenträger der Kirche wie Papst Johannes Paul II. sehr wohl mit den Inszenierungsmöglichkeiten einer „populären Religion“ (279) und Musik spielen. M. Fuhr bringt eine ganz neue Perspektive zur Sprache: Ihm geht es weniger um die Spielarten der populären Musik als um grundsätzliche Strategien der Exklusion in der abendländischen Musikkritik, die lange Zeit die Autonomie-Ästhetik als ihre Grundlage betrachtete. Individuelle Schöpfung, Werkcharakter, Balance von Geist und Sinnlichkeit etc. standen der ,Primitivität' des ,Anderen' gegenüber, dem also, was als außereuropäische Musik bezeichnet wurde und bestenfalls durch einen evolutionären Prozess ,gerettet' werden konnte. Mit den 1950er- und 1960er Jahren erfolgte sodann bereits vor dem Cultural Turn der Birminghamer Schule der ,cultural turn' der Musikwissenschaft, der zur Ablöse des eurozentrischen Musikbegriffs der Vergleichenden Musikwissenschaft durch den „kulturalistischen und kulturrelativistischen“ (301) einer pluralistischen Musikethnologie führte. Sie ist „engagierte Produzentin von Differenz und Kulturkritik“ (301), eine Kulturkritik, die nunmehr ,viele Orte“ kennt (301).

Die beiden letzten Beiträge des Sammelbandes nehmen zwei konkrete kulturelle bzw. kulturpolitische Ereignisse ins Visier, um auf ihre Weise das Zusammenspiel von Kulturkritik und Populärem in der Musik zu charakterisieren. Anhand des Songs „Mussolini“ des der Neuen Deutschen Welle (Punk, New Wave) nahestehenden Duos DAF zeigt B. Hornberger, dass „,irritierende Artefakte“ nur in ihrer „spezifischen kulturellen Einbettung“ verstanden werden können (317). So setze „Mussolini“ mit Verrätselung, Provokation und der Verweigerung einer klaren Positionierung auf „Faschismus als Rotes Tuch für die Generation, die sich abarbeiten musste an der NS-Vergangenheit der eigenen Väter und deren Verdrängung der Schuld nach dem Krieg“ (317). Die (von K. Passig 2009 benannten) „Standardsituationen der Technologiekritik" allein - ästhetische Minderwertigkeit, ideologische Zweifelhaftigkeit, Gefährdung der Jugend und Kommerzialität/Kalkül (306-316) - greifen hier zu kurz. „Vom Popmusikmanager zum Kulturstaatssekretär!?“ (321) aus der Feder von S. Binas-Preisendörfer analysiert schlussendlich den Pressediskurs rund um die Bestellung von Tim Renner, dem ehemaligen Geschäftsführer der Motor Entertainment GmbH, zum Staatssekretär für Kulturelle Angelegenheiten in Berlin (2014) und zeigt, dass selbst wenn die aktuelle Kulturpolitik auf gesellschaftliche Veränderungen reagiert, diese neue Art von Kulturverständnis den Erwartungen „kultureller Eliten“ (331) immer noch entgegenläuft. Zwei Jahre später - im Dezember 2016 - bleibt somit abzuwarten, wie diese Eliten die soeben erfolgte Ernennung von Bogdan Roscic, dem ehemaligen Musikchef von Ö3, zum Direktor der Wiener Staatsoper kommentieren werden...

Zum Fazit: 337 Seiten überwiegend dichter Beiträge, zwei davon in englischer Sprache verfasst, machen den vorliegenden Sammelband zu einem wichtigen Wegweiser für den Umgang mit dem ,Populären in der Musik' bzw. der ,Populärmusik'. Die Beiträge überzeugen und sind - mit einigen wenigen Ausnahmen - trotz der Komplexität der Materie 
gut lesbar. Was auf den ersten Blick wie eine Fülle unterschiedlichster Ansätze wirken mag, fügt sich bald schon zu einem Ganzen, das auch kritische Perspektiven beinhaltet bzw. den Grundstein legt für den weiterführenden Umgang mit bekannten Positionen. Dem Herausgeber ist damit eine sehr gute Publikation gelungen.

Ursula MATHIS-MOSER (Innsbruck) 